PROCEEDINGS OF THE

AMERICAN MATHEMATICAL SOCIETY

Volume 131, Number 10, Pages 3135-3141

S 0002-9939(03)06883-7

Article electronically published on January 2, 2003

\title{
AN APPLICATION OF THE PUTNAM-FUGLEDE THEOREM TO NORMAL PRODUCTS OF SELF-ADJOINT OPERATORS
}

\author{
HICHEM M. MORTAD
}

(Communicated by Joseph A. Ball)

\begin{abstract}
We prove that if we have two self-adjoint operators (bounded or not) and if their product is normal, then it is self-adjoint provided a certain condition is satisfied.
\end{abstract}

\section{INTRODUCTION}

In 2000 E. Albrecht and P. G. Spain [1] proved that if we have two bounded selfadjoint operators $K, H$ and if $K$ satisfies $\sigma(K) \cap \sigma(-K) \subseteq\{0\}$ (we shall call this condition on the spectrum of $K$ condition C), then $H K$ normal implies $H K$ selfadjoint. The proof was given in a more general context of Banach algebras, hence the result in $\mathcal{B}(H)$ was just a consequence of the main theorem in that paper. However, nothing was said about the case when at least one of the operators is unbounded. In this paper we answer this question positively, i.e. if $K$ is a bounded self-adjoint operator satisfying condition $\mathrm{C}$ and if $H$ is any unbounded self-adjoint operator, then the result holds. Even when both $K$ and $H$ are unbounded selfadjoint operators such that $K$ satisfies condition $\mathrm{C}$, the result also holds.

In the end we give a counterexample that shows that the product of two unbounded self-adjoint operators, when it has a normal closure, is not necessarily essentially self-adjoint (here the notion of essentially self-adjoint means that the operator has a self-adjoint closure) even when condition $\mathrm{C}$ is satisfied.

We assume basic definitions and notions about unbounded operators. For this literature see [5] or [2]. The operators dealt with are defined either on a Hilbert space or on a dense domain of a Hilbert space depending on their boundedness.

We also note that one can prove the result of Albrecht-Spain without calling on the theory of Banach algebras which was used in [1]. The proof is given below.

\section{Results}

We recall the Albrecht-Spain theorem:

Theorem 1. Let $H$ and $K$ be two bounded self-adjoint operators. Let $K$ satisfy condition $\mathrm{C}$. If $\mathrm{HK}$ is normal, then it is self-adjoint.

Proof. We have $K H K=K N=N^{*} K$. Then using the Fuglede theorem we obtain $K N^{*}=N K$ or $K^{2} H=H K^{2}$ and then because $f: \sigma\left(K^{2}\right) \rightarrow \sigma(K): \lambda^{2} \mapsto \lambda$

Received by the editors March 8, 2002 and, in revised form, April 30, 2002.

2000 Mathematics Subject Classification. Primary 47B15, 47B25.

(C)2003 American Mathematical Society 
is well-defined and continuous, $f\left(K^{2}\right) H=H f\left(K^{2}\right)$ or $K H=H K$, which implies that $H K$ is self-adjoint.

Remark 1. It is easy to construct noncommuting self-adjoint operators $H$ and $K$ with $H^{2}=K^{2}=I$, so some additional condition is required to get $H K=K H$ from the fact that $H K^{2}=K^{2} H$. This is accomplished by condition C.

We also recall the Fuglede-Putnam theorem [4]:

Theorem 2. If $N, M$ are two densely defined normal operators and if $A$ is a bounded operator such that $A N \subset M A$, then $A N^{*} \subset M^{*} A$.

Definition 1. Let $K$ be a bounded operator and $H$ an unbounded one. Then $K$ and $H$ are said to commute if $K H \subset H K$.

Proposition 1. Let $K$ be a bounded self-adjoint operator and let $H$ be an unbounded self-adjoint one such that both $K$ and $H$ commute. Then for any continuous function $f$ defined on the compact set $\sigma(K)$ we also have that $f(K)$ and $H$ commute.

Proof. Before we start the proof we need the following lemma:

Lemma 1. If $K$ and $H$ commute where $K$ is self-adjoint, then for any real polynomial $P, P(K)$ and $H$ also commute.

Proof. Set $P(\lambda)=a_{0}+a_{1} \lambda+\ldots+a_{n} \lambda^{n}$ (the coefficients being real). Let $x \in$ $\mathcal{D}(H)=\mathcal{D}(P(K) H)=\mathcal{D}(K H)=\mathcal{D}\left(K^{2} H\right)=\ldots=\mathcal{D}\left(K^{n} H\right)$. $K, H$ commute so $K H \subset H K$, i.e. $K H x=H K x$ for all $x \in \mathcal{D}(K H)$ and $\mathcal{D}(K H) \subset \mathcal{D}(H K)$. Also $K^{2} H=K(K H) \subset K(H K)=(K H) K \subset H K^{2}$, i.e. $K^{2} H x=H K^{2} x$ for all $x$ in $\mathcal{D}\left(K^{2} H\right)=\mathcal{D}(H)$ and $\mathcal{D}\left(K^{2} H\right) \subset \mathcal{D}\left(H K^{2}\right)$.

We do the same to the powers of $K$ until we get $K^{n} H \subset H K^{n}$, i.e. $K^{n} H x=$ $H K^{n} x \forall x \in \mathcal{D}\left(K^{n} H\right)$ and $\mathcal{D}\left(K^{n} H\right) \subset \mathcal{D}\left(H K^{n}\right)$. Hence $\forall x \in \mathcal{D}(P(K) H)=\mathcal{D}(H)$ we have $\left(a_{0} I H+a_{1} K H+a_{2} K^{2} H+\ldots+a_{n} K^{n} H\right) x=\left(H a_{0} I+H a_{1} K+H a_{2} K^{2}+\ldots+\right.$ $\left.H a_{n} K^{n}\right) x$ and $\mathcal{D}(P(K) H) \subset \mathcal{D}(H P(K))$. This shows that $P(K)$ and $H$ commute, i.e. $P(K) H \subset H P(K)$.

As the set of polynomials (that are defined on a compact set; here it is $\sigma(K)$ ) is dense in the set of continuous functions we can say that there is a sequence of polynomials $P_{n}$ s.t. $P_{n} \rightarrow f$ in the supremum norm on $\sigma(K)$.

This implies that $P_{n}(K) \rightarrow f(K)$ in $\mathcal{L}(H)$. Let $y \in \mathcal{D}(H)$. Set $x_{n}=P_{n}(K) y$ and $x=f(K) y$. We have $H x_{n}=H P_{n}(K) y=P_{n}(K) H y \rightarrow f(K) H y . H$ is closed and $x_{n} \rightarrow x$ imply that $f(K) y \in \mathcal{D}(H)$ and $H x=f(K) H y$, i.e. $f(K) H \subset H f(K)$.

Remark 2. One only needs the closedness of $H$ in this lemma.

Theorem 3. Let $H$ be a densely defined self-adjoint operator and let $K$ be a bounded self-adjoint operator such that $\sigma(K) \cap \sigma(-K) \subseteq\{0\}$. If $H K$ is normal, then it is self-adjoint.

Proof. $N=H K$ is normal. We know that $N^{*}=(H K)^{*} \supset K^{*} H^{*}=K H$.

We have

$$
K H K=(K H) K=K(H K) \Rightarrow K N=(K H) K \subset N^{*} K .
$$

But $N$ and $N^{*}$ are both normal, so by means of the Fulglede-Putnam theorem we get $K N^{*} \subset N^{* *} K=\bar{N} K=N K$ since $N$ is closed. 
It follows that $K^{2} H=K(K H) \subset K N^{*} \subset N K=(H K) K=H K^{2}$, i.e. $K^{2}$ and $H$ commute in the sense of the definition given above. Now the function $f: \sigma\left(K^{2}\right) \rightarrow \sigma(K), \lambda^{2} \mapsto \lambda$ is well-defined thanks to condition C. Besides, $f$ is continuous. This implies that $f\left(K^{2}\right)$ and $H$ commute or $K$ and $H$ commute, i.e. $K H \subset H K$,

$$
K H \subset H K \Rightarrow(H K)^{*} \subset(K H)^{*}=H^{*} K^{*}=H K .
$$

Since $H K$ is normal, then $\mathcal{D}(H K)=\mathcal{D}\left((H K)^{*}\right)$ and on $D\left((H K)^{*}\right)$ we have $(H K)^{*}=H K$ which shows that $H K$ is self-adjoint.

Theorem 4. Under the same assumptions as Theorem 3 and instead of assuming that $H K$ is normal we assume that $K H$ is normal. Then $K H$ is self-adjoint.

Proof. If $K H$ is normal, then so is $(K H)^{*}$. But $(K H)^{*}=H K$, i.e. $H K$ is normal. So as a consequence of Theorem 1 we know that $H K$ is self-adjoint, i.e. $(H K)^{*}=$ $H K$.

On the other hand $(K H)^{*}=H K$ so that $(K H)^{*}$ is self-adjoint, i.e. $(K H)^{* *}=$ $(K H)^{*}$ but $(K H)^{* *}=\overline{K H}=K H$ as $K H$ is closed (it is normal). Thus $K H=$ $(K H)^{*}$, i.e. $K H$ is self-adjoint.

Corollary 1. Let $K$ be a bounded positive self-adjoint operator and let $H$ be any unbounded self-adjoint operator. Then if $H K$ is normal (resp. KH is normal), it is self-adjoint (resp. it is self-adjoint).

Now the case where both $K$ and $H$ are unbounded. The result is also true. Besides, one has a generalization of the Fuglede-Putnam theorem with rather stronger conditions.

Theorem 5. If $N$ is an unbounded normal operator and if $K$ is a self-adjoint one such that $D(N) \subset D(K)$, then $K N \subset N^{*} K$ implies $K N^{*} \subset N K$.

Proof. Let us consider the ball $B_{R}=\{z \in \mathbf{C}|| z \mid \leq R\}$. Let $P_{B_{R}}$ be the spectral projection for $N$ defined on the Borel set $B_{R} . N$ has the following spectral decomposition: $N=\int_{\sigma(N)} \lambda d P_{\lambda}$. One can show that

$$
f \in \operatorname{RanP}_{B_{R}} \Leftrightarrow \exists c>0 \text { such that }\left\|N^{k} f\right\| \leq c R^{k}, \forall k=1,2, \ldots .
$$

It does not matter whether $N$ or $N^{*}$ is inside the norm. Let us restrict $K$ to the Hilbert space $\operatorname{Ran} P_{B_{R}}$ (in the sequel $\operatorname{Ran} P_{B_{R}}$ will be denoted by $H_{R}$ ). We claim that $K: H_{R} \rightarrow H_{R}$ and that $K$ is bounded. $H_{R}$ is a subset of $\mathcal{D}(K)$ since $H_{R} \subset \mathcal{D}(N)$ by the spectral theorem and $\mathcal{D}(N) \subset \mathcal{D}(K) . K$ is bounded as it is defined everywhere and its graph is closed. The boundedness of $K$ then follows from the closed graph theorem. Let $\varphi \in H_{R}$. From (1) we have $K \varphi \in H_{R}$ if and only if $\left\|\left(N^{*}\right)^{k} K \varphi\right\| \leq \alpha R^{k}$. We also have $\left\|N^{k} \varphi\right\| \leq c R^{k}$ so that $\left\|K N^{k} \varphi\right\| \leq \alpha R^{k}$, but for such $\varphi$ we have $\left\|K N^{k} \varphi\right\|=\left\|\left(N^{*}\right)^{k} K \varphi\right\|$ as a consequence of the hypothesis in the theorem and hence $K \varphi \in H_{R}$. Now let $\varphi \in \mathcal{D}\left(K N^{*}\right)$. Then $\exists \varphi_{n} \in H_{n}$ such that $\varphi_{n} \rightarrow \varphi$. We can choose $\varphi_{n}=P_{B_{n}} \varphi \cdot \varphi_{n}$ is in $\mathcal{D}\left(K N^{*}\right)$ since $K N^{*}$ is bounded on $H_{n}$. We need to show that $K N^{*} \varphi_{n} \rightarrow K N^{*} \varphi$. Since $K: H_{R} \rightarrow H_{R}$ then $K P_{B_{R}}=P_{B_{R}} K$ as $H_{R}$ reduces $K$. We now have $K N^{*} \varphi_{n}=P_{B_{n}} K N^{*} \varphi \rightarrow K N^{*} \varphi$.

We now need to show that $N K \varphi_{n} \rightarrow N K \varphi$. The operator $K N_{/ H_{n}}^{*}$ is bounded because both $N^{*}$ and $K$ are so on $H_{n}$. On $H_{n}$ we have $K N \varphi_{n}=N^{*} K \varphi_{n}$. Using the Fuglede theorem for the bounded operators we get $K N^{*} \varphi_{n}=N K \varphi_{n}$, hence $N K \varphi_{n} \rightarrow K N^{*} \varphi$. 
$N$ maps $H_{R}^{\perp}=\operatorname{Ran} P_{B_{R}^{c}}$ to $H_{R}^{\perp}\left(H_{R}\right.$ is a reducing space for $\left.N\right)$ and $N^{-1}$ is bounded on $H_{R}{ }^{\perp}$ since in this case $N^{-1}=\int_{B_{R}^{c}} \frac{1}{\lambda} d P_{\lambda}$ and hence $\left|\frac{1}{\lambda}\right| \leq \frac{1}{R}$.

We also have $N K \varphi_{n}-K N^{*} \varphi=K N^{*} \varphi_{n}-K N^{*} \varphi \in H_{R}^{\perp}$ for $n>R$ so that if we apply the inverse of $N$ we get $K \varphi_{n} \rightarrow N^{-1} K N^{*} \varphi$, and $K$ being closed we obtain $K \varphi_{n} \rightarrow K \varphi . \quad N$ is closed and $\left(N K \varphi_{n}\right)_{n}$ convergent together with $K \varphi_{n} \rightarrow K \varphi$ imply $N K \varphi_{n} \rightarrow N K \varphi$, i.e. $\varphi \in \mathcal{D}(N K)$.

We also have on $\mathcal{D}\left(K N^{*}\right), K N^{*} \varphi \leftarrow K N^{*} \varphi_{n}=N K \varphi_{n} \rightarrow N K \varphi$. Hence by uniqueness of the limit $K N^{*} \varphi=N K \varphi$ on $\mathcal{D}\left(K N^{*}\right)$, i.e. $K N^{*} \subset N K$.

Corollary 2. Let $K, H$ be two unbounded self-adjoint operators. If $N=H K$ is normal, then $K N \subset N^{*} K$ implies $K N^{*} \subset N K$.

Proof. Obvious since $\mathcal{D}(N)=\mathcal{D}(H K) \subset \mathcal{D}(K)$.

Theorem 6. Let $K, H$ be two unbounded self-adjoint operators such that $\sigma(K) \cap$ $\sigma(-K) \subseteq\{0\}$. If $H K$ is normal, then it is self-adjoint.

Proof. Set $N=H K$. We have $K H K=K(H K)=(K H) K \subset(H K)^{*} K$ which implies that $K N \subset N^{*} K$ but $\mathcal{D}(N) \subset \mathcal{D}(K)$. Then from Corollary 2 we can say that $K N^{*} \subset N K$ or $K^{2} H \subset K(H K)^{*} \subset H K^{2}$. We have $K^{2} H \varphi=H K^{2} \varphi$ for $\varphi \in \mathcal{D}\left(K^{2} H\right)$. Using the same arguments as in the proof of Theorem 5 we can say that for $\varphi \in \operatorname{Ran}_{B_{n}}$ we have $K^{2} H K \varphi=H K^{2} K \varphi$ as $K \varphi \in \mathcal{D}\left(K^{2} H\right)$ since $K^{2} N$ is bounded in this case. We have $K^{2} N \varphi=N K^{2} \varphi$.

Now take the same function $f$ taken in the proof of Theorem 3 to get $f\left(K^{2}\right) N \varphi=$ $N f\left(K^{2}\right) \varphi$ and hence $K N \varphi=N K \varphi$. But $K N \varphi=N^{*} K \varphi$ on $H_{n}$. Hence $N^{*} K \varphi=$ $N K \varphi$.

We now use the orthogonal decomposition $H_{n}=\overline{\operatorname{RanK}} \bigoplus \operatorname{Ker} K$ for the $K$ restricted to $H_{n}$. We have $N=N^{*}$ on $\overline{\operatorname{RanK}}$ and both 0 on $\operatorname{Ker} K$.

Hence $N=N^{*}$ on $H_{n}$. This shows that $N_{n}\left(N_{n}\right.$ is just $N$ restricted to $\left.H_{n}\right)$ is self-adjoint. Hence $\sigma\left(N_{n}\right) \subseteq \mathbf{R}$ for all $n$ and then $\sigma(N) \subseteq \mathbf{R}$ and a normal operator with a real spectrum is self-adjoint. Thus $H K$ is self-adjoint.

Corollary 3. Let $K, H$ be two densely defined self-adjoint operators such that $K$ is positive. If $H K$ is normal, then it is self-adjoint.

Remark 3 . We have seen that the result is true for any couple of self-adjoint operators regardless of their boundedness and provided condition $\mathrm{C}$ is satisfied. However, the hypothesis " $H K$ normal" cannot be replaced by " $H K$ having a normal closure". Here we give a counterexample.

\section{A counterexample}

Let us consider the operators $K$ and $H$ defined as

$$
H=-i \frac{d}{d x}: H^{1}(\mathbf{R}) \rightarrow L^{2}(\mathbf{R}), K=|x|: \mathcal{D}(K) \rightarrow L^{2}(\mathbf{R})
$$

where $\mathcal{D}(K)=\left\{f \in L^{2}(\mathbf{R}) \| x \mid f \in L^{2}(\mathbf{R})\right\}$ and $H^{1}(\mathbf{R})$ denotes the Sobolev space $\left\{f \in L^{2}(\mathbf{R}) \mid f^{\prime} \in L^{2}(\mathbf{R})\right\}$. $K$ is obviously positive so that it does satisfy condition C. We also know that those two operators are self-adjoint on the given domains. $N=H K$ is defined on $\mathcal{D}(H K)$, that is,

$$
\{f \in \mathcal{D}(K) \mid K f \in \mathcal{D}(H)\}=\left\{f \in L^{2}(\mathbf{R}) \| x \mid f,-i(|x| f)^{\prime} \in L^{2}(\mathbf{R})\right\}
$$

such that $N f=-i(|x| f)^{\prime}$ where the derivative is taken in the distributional sense. 
The operator $N$ is densely defined since it contains $C_{0}^{\infty}(\mathbf{R})$ (the space of $C^{\infty}$ functions that have a compact support). It is not closed but it has a closed extension $\bar{N}$ defined on $\mathcal{D}(\bar{N})$, which consists of the $L^{2}$-functions s.t. $|x| f^{\prime}$ is in $L^{2}(\mathbf{R})$ where $|x| f^{\prime}$ is a distribution on $\mathbf{R} \backslash\{0\}$, by $\bar{N} f=-i|x| f^{\prime} \pm i f$.

We need to check that $\bar{N}$ is closed on this domain with respect to the graph norm of $\bar{N}$.1 Take $\left(f_{n}, \bar{N} f_{n}\right) \in \mathcal{G}(\bar{N})$ such that $\left(f_{n}, \bar{N} f_{n}\right) \rightarrow(f, g) . f_{n} \rightarrow f$, then in the distributional sense we have $f_{n}^{\prime} \rightarrow f^{\prime}$. On $\mathbf{R} \backslash\{0\}$ we have $|x| f_{n}^{\prime} \rightarrow|x| f^{\prime}$. By uniqueness of the limit $g=|x| f^{\prime}$ for almost every $x$, hence we have the equality in $L^{2}(\mathbf{R})$. This tells us that $\bar{N}$ is closed in this domain.

The operator $\bar{N}$ is a closed extension of $N$. It is in fact the closure of $N$, and this will be shown once we have shown that $C_{0}^{\infty}(\mathbf{R} \backslash\{0\})$ is dense in $\mathcal{D}(\bar{N})$ with respect to the graph norm of $\bar{N}$.

The set of the functions in $\mathcal{D}(\bar{N})$ that have compact support that is away from the origin will be denoted by $\mathcal{D}(\bar{N})_{\Omega^{*}}$.

Lemma 2. $C_{0}^{\infty}(\mathbf{R} \backslash\{0\})$ is dense in $\mathcal{D}(\bar{N})_{\Omega^{*}}$ with respect to the graph norm of $\bar{N}$.

Proof. Let $f$ be in $\mathcal{D}(\bar{N})_{\Omega^{*}}$. Let us find a sequence $f_{n}$ in $C_{0}^{\infty}(\mathbf{R} \backslash\{0\})$ s.t. $f_{n} \rightarrow f$ in the graph norm of $\bar{N}$, that is,

$$
0 \leftarrow\left\|f_{n}-f\right\|_{\mathcal{D}(\bar{N})}^{2}=\left\|f_{n}-f\right\|_{2}^{2}+\left\|x f_{n}^{\prime}-x f^{\prime}\right\|_{2}^{2} .
$$

It suffices to show that the right-hand side converges to zero as $n$ tends to infinity. We know that if $f$ lies in the space $L_{\Omega^{*}}^{2}$ of $L^{2}$ functions of compact support that is away form the origin, then $k_{n} * f \in C_{0}^{\infty}$ for all $n$ in $\mathbf{N}$ and

$$
\lim _{n \rightarrow \infty}\left\|f-k_{n} * f\right\|_{2}=0
$$

where $k_{n}$ is a sequence of smooth functions that have compact support away from the origin defined by $k_{n}(x)=n k(n x)$ where $k$ is a $C_{0}^{\infty}$ function with support away from the origin such that $\int_{\mathbf{R}} k(x) d x=1$.

Now take $f_{n}=k_{n} * f$ which is in $C_{0}^{\infty}(\mathbf{R} \backslash\{0\})$ if $f$ has the support $\Omega^{*}$. We need to check that $f_{n} \rightarrow f$ in the graph norm of $\bar{N}$. The convergence of $f_{n}$ to $f$ in the $L^{2}$-norm follows from (2).

We have $f^{\prime} \in L_{\Omega^{*}}^{2}$ as we have $|x| f^{\prime} \in L_{\Omega^{*}}^{2}$. We also have $f_{n}^{\prime}=k_{n} * f^{\prime}$ (in the distributional sense) so that (1) gives $\left\|x\left(k_{n} * f^{\prime}\right)-x f^{\prime}\right\|_{2} \rightarrow 0$, hence $\left\|f_{n}-f\right\|_{\mathcal{D}(\bar{N})} \rightarrow$ 0 as $n \rightarrow \infty$. This shows that $C_{0}^{\infty}(\mathbf{R} \backslash\{0\})$ is dense in $\mathcal{D}(\bar{N})_{\Omega^{*}}$.

Lemma 3. $\mathcal{D}(\bar{N})_{\Omega^{*}}$ is dense in $\mathcal{D}(\bar{N})$.

Proof. Let us find a sequence $f_{n}$ in $\mathcal{D}(\bar{N})_{\Omega^{*}}$ and let us show that $f_{n} \rightarrow f$ in the graph norm of $\bar{N}$. Take $f_{n}=f . \varphi_{n}$ where

$$
\varphi_{n}(x)= \begin{cases}n\left(x-\frac{1}{n}\right) & \text { if } \frac{1}{n} \leq x<\frac{2}{n} \\ 1 & \text { if } \frac{2}{n} \leq x<n \\ -\frac{1}{n}(x-n)+1 & \text { if } n \leq x \leq 2 n \\ 0 & \text { otherwise. }\end{cases}
$$

\footnotetext{
${ }^{1}$ The graph norm in this case is defined as $\|f\|_{\mathcal{D}(\bar{N})}^{2}=\|f\|_{L^{2}(\mathbf{R})}^{2}+\left\||x| f^{\prime}\right\|_{L^{2}(\mathbf{R})}^{2}$. It should have been $\|f\|_{\mathcal{D}(\bar{N})}^{2}=\|f\|_{L^{2}(\mathbf{R})}^{2}+\left\||x| f^{\prime} \pm f\right\|_{L^{2}(\mathbf{R})}^{2}$, but the two norms are equivalent.
} 


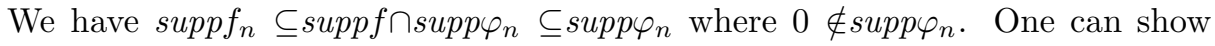
that $\varphi_{n}$ tends to 1 pointwise. Also $\varphi_{n}^{\prime}$ exists almost everywhere. We need to show that $f_{n} \rightarrow f$ in the graph norm of $\bar{N}$.

$$
\left\|f_{n}-f\right\|_{L^{2}(\mathbf{R})}^{2}=\int_{\mathbf{R}}\left|f_{n}(x)-f(x)\right|^{2} d x=\int_{\mathbf{R}}|f(x)|^{2}\left(\varphi_{n}(x)-1\right)^{2} d x \rightarrow 0
$$

by the D.C.T. (dominated convergence theorem).

We also have $f_{n}^{\prime}(x)=f^{\prime}(x) \varphi_{n}(x)+f(x) \varphi_{n}^{\prime}(x)$. Then

$$
\begin{aligned}
\left\|x f_{n}^{\prime}-x f^{\prime}\right\|_{L^{2}(\mathbf{R})}^{2} & =\int_{\mathbf{R}}\left|x f_{n}^{\prime}(x)-x f^{\prime}(x)\right|^{2} d x \\
& \leq 2 \int_{\mathbf{R}}\left|x f^{\prime}(x)\right|^{2}\left(\varphi_{n}(x)-1\right)^{2} d x+2 \int_{\mathbf{R}}\left|x f(x) \varphi_{n}^{\prime}(x)\right|^{2} d x .
\end{aligned}
$$

The first bit of the integral tends to zero again by the D.C.T. (the dominating function being $\left.\left(x f^{\prime}\right)^{2} \in L^{1}(\mathbf{R})\right)$. For the second bit we have $\int_{\mathbf{R}} x^{2}|f(x)|^{2}\left|\varphi_{n}^{\prime}(x)\right|^{2} d x=$ $\int_{\frac{1}{n}}^{\frac{2}{n}} x^{2} n^{2}|f(x)|^{2} d x+\int_{n}^{2 n} \frac{x^{2}}{n^{2}}|f(x)|^{2} d x$.

We have $\int_{\frac{1}{n}}^{\frac{2}{n}} x^{2} n^{2}|f(x)|^{2} d x \leq 4 \int_{\frac{1}{n}}^{\frac{2}{n}}|f(x)|^{2} d x=4 \int_{\mathbf{R}}|f(x)|^{2} \mathbf{1}_{\left[\frac{1}{n}, \frac{2}{n}\right]}(x) d x \rightarrow 0$ by the D.C.T. since $\lim _{n \rightarrow \infty} \mathbf{1}_{\left[\frac{1}{n}, \frac{2}{n}\right]}(x)=0$.

We also have $\int_{n}^{2 n} \frac{x^{2}}{n^{2}}|f(x)|^{2} d x \leq 4 \int_{n}^{2 n}|f(x)|^{2} d x=4 \int_{\mathbf{R}}|f(x)|^{2} \mathbf{1}_{[n, 2 n]}(x) d x$ which tends to 0 by the D.C.T. Thus $\left\|x f_{n}^{\prime}-x f^{\prime}\right\|_{L^{2}(\mathbf{R})}^{2} \rightarrow 0$. This tells us that $\left\|f_{n}-f\right\|_{\mathcal{D}(\bar{N})} \rightarrow 0$.

$C_{0}^{\infty}(\mathbf{R} \backslash\{0\})$ is dense in $\mathcal{D}(\bar{N})_{\Omega^{*}}$ and the latter is dense in $\mathcal{D}(\bar{N})$. Therefore $C_{0}^{\infty}(\mathbf{R} \backslash\{0\})$ is dense in $\mathcal{D}(\bar{N})$ w.r.t. the graph norm of $\bar{N}$.

Corollary 4. $\bar{N}$ is the closure of $N$.

Proof. This follows from $C_{0}^{\infty}(\mathbf{R} \backslash\{0\}) \subset \mathcal{D}(N) \subset \mathcal{D}(\bar{N})$. Hence $\mathcal{D}(N)$ is dense in $\mathcal{D}(\bar{N})$ w.r.t. the graph norm of $\bar{N}$.

In order to find the adjoint of $N$ on $\mathcal{D}(N)$ it suffices to find it on $C_{0}^{\infty}(\mathbf{R} \backslash\{0\})$. If we restrict $N$ to $C_{0}^{\infty}(\mathbf{R} \backslash\{0\})$ and we denote it by $N_{0}$, then it is known that $N^{*}=N_{0}^{*}$. The domain of $N^{*}$ is defined as

$$
\mathcal{D}\left(N^{*}\right)=\left\{g \in L^{2}(\mathbf{R}) \mid \exists h \in L^{2}(\mathbf{R}) \text { s.t. }\langle N f, g\rangle=\langle f, h\rangle \forall f \in C_{0}^{\infty}(\mathbf{R} \backslash\{0\})\right\},
$$

and we have

Lemma 4. $\mathcal{D}\left(N^{*}\right)=\left\{f \in L^{2}(\mathbf{R}) \| x \mid f^{\prime} \in L^{2}(\mathbf{R})\right\}$.

Remark 4 . We denote a distribution $T$ by $(T, \varphi)$ where $\varphi$ is a $C_{0}^{\infty}$ function.

Proof. Let $f \in C_{0}^{\infty}(\mathbf{R} \backslash\{0\})$ and $g \in L^{2}(\mathbf{R})$. We have

$$
\langle N f, g\rangle=\int_{\mathbf{R}}(|x| f(x) \mid)^{\prime} \overline{i g(x)} d x=\left((|x| f)^{\prime}, \overline{i g}\right)
$$

since $\left.(|x| f)^{\prime} \in C_{0}^{\infty}(\mathbf{R} \backslash\{0\})\right)$. By definition of the distributional derivative,

$$
\left((|x| f)^{\prime}, \overline{i g}\right)=-\left(|x| f,-i \bar{g}^{\prime}\right)=\left(f, i|x| \bar{g}^{\prime}\right)
$$

by definition of the product of distributions since $|x|$ is $C^{\infty}$ on $\mathbf{R} \backslash\{0\}$. We also have $\langle f, h\rangle=(f, \bar{h})$. Hence $h=-i|x| g^{\prime}$ as a distribution but $h$ is in $L^{2}$. Then $|x| g^{\prime} \in L^{2}$ and then $\mathcal{D}\left(N^{*}\right)=\left\{g \in L^{2}|| x \mid g^{\prime} \in L^{2}\right\}$ and $N^{*} g=-i|x| g^{\prime}$. 
It is known that $\mathcal{D}\left(N^{*}\right)=\mathcal{D}\left(\bar{N}^{*}\right)$. Clearly $\bar{N}$ is not self-adjoint (it is not even symmetric as $\left.\bar{N}-\bar{N}^{*} \subseteq \pm i\right)$. However, it is normal as

$$
\bar{N} \cdot \bar{N}^{*} f(x)=\bar{N}\left(-i|x| f^{\prime}(x)\right)=-i\left(-i|x||x| f^{\prime}(x)\right)^{\prime}=-x^{2} f^{\prime \prime}(x)-2 x f^{\prime}(x)
$$

and

$$
\bar{N}^{*} \cdot \bar{N} f(x)=\bar{N}^{*}\left[-i(|x| f(x))^{\prime}\right]=-x^{2} f^{\prime \prime}(x)-2 x f^{\prime}(x) .
$$

We also have

$$
\mathcal{D}\left(\bar{N} \cdot \bar{N}^{*}\right)=\left\{f \in \mathcal{D}\left(\bar{N}^{*}\right) \mid \bar{N}^{*} f \in \mathcal{D}(\bar{N})\right\}=\left\{f \in L^{2}(\mathbf{R})|,| x \mid f^{\prime}, x^{2} f^{\prime \prime} \in L^{2}(\mathbf{R})\right\}
$$

and $\mathcal{D}\left(\bar{N}^{*} \cdot \bar{N}\right)$ is exactly the same.

Thus, we have found two unbounded self-adjoint operators $H, K$ such that $\sigma(K) \cap$ $\sigma(-K) \subseteq\{0\}$ for which $N=H K$ has a normal closure without being essentially self-adjoint.

\section{ACKNowledgement}

I owe many thanks to my supervisor, Professor A.M. Davie, for the help he has given me. I also thank the Algerian Government for the full scholarship it has granted me.

\section{REFERENCES}

1. E. Albrecht, P. G. Spain, When Products of Selfadjoints Are Normal, Proc. American Math. Soc., 128/8 (2000) 2509-2511. MR 2000m:46001

2. J. B. Conway, A Course in Functional Analysis, Springer, 1990 (2nd edition). MR 91e:46001

3. B. Fuglede, A Commutativity Theorem For Normal Operators, Proc. National Acad. Sci., 36 (1950) 35-40. MR 13:253d

4. C. R. Putnam, On Normal Operators in Hilbert Space, Amer. J. Math., 73 (1951) 357-362. MR 12:717f

5. M. Reed, B. Simon, Methods of Modern Mathematical Physics (Vol.1, Functional Analysis), Acad. Press, 1972. MR 58:12429a

School of Mathematics, University of Edinburgh, JCMB, Mayfield Road, Edinburgh, EH9 3JZ, United Kingdom

E-mail address: mortad@maths.ed.ac.uk 